Proceedings of the 1996 IEEE

International Conference on Robotics and Automation

Minneapolis, Minnesota - April 1996

\title{
Passive robots and haptic displays based on nonholonomic elements
}

\author{
Michael Peshkin \\ J. Edward Colgate \\ Carl Moore \\ Mechanical Engineering Dept. \\ Northwestern University \\ Evanston IL 60208
}

\begin{abstract}
We describe a new architecture for passive robots and haptic displays, which we call a programmable constraint machine (PCM). An n-dof PCM can, under computer control, exhibit constraints (smooth, impenetrable virtual surfaces of dimensionality $<n$ ), or it can allow free $n$-dof motion.

At the heart of the PCM is a nonholonomic element, which is used as a continuously variable transmission (CVT). A rolling wheel, for instance, can be used as a CVT. A prototype 2-dof cartesian PCM has been built, using a single rolling wheel, We sketch PCMs of higher dimensionality.

A rolling wheel may be thought of as a translational CVT, coupling the $x$ and $y$ velocities of its center by $a$ transmission ratio which is the tangent of its steering angle, Its utility in a cartesian PCM motivates interest in a rotational analog for revolute architectures. We develop a novel rotational CVT which couples two angular velocities by an adjustable ratio.
\end{abstract}

\section{Motivation}

Several recent areas of interest in robotics require the direct physical interaction of people with manipulators, haptic displays, or assistive devices. For example, in computer assisted surgery a surgeon may move a surgical tool cooperatively with a robot, with the robot enforcing certain constraints (e.g. "cut only on this surface") while the surgeon is free to maneuver the tool within the allowed surface. In virtual reality applications, a user may interact with and "feel" objects which exist only in digital representation, by using a haptic display to probe those virtual objects and perceive the resulting reaction forces. In vehicle final assembly, workers may cooperatively control the motion of large heavy components with an assistive device, where the assistive device controls some aspects or bounds on its motion, while the worker controls others.

The above examples have in common the exchange of force and motion between a human and a multi-dof machine. Different requirements apply to robots such as these, that interact energetically with people, than apply to industrial robots in a typical humans-excluded environment. First, the development of perceptually smooth force-following, in which the robot is guided by the human user, has been found to be quite difficult, and to require much higher servo rates than are needed for position control alone. Second, safety becomes a critical issue, since a robot with a payload of only a few pounds can quickly develop a lethal kinetic energy. Finally, humans present an unpredictable impedance, and guaranteeing stability of a robot in physical interaction with a person is non-trivial [Colgate \& Brown, 1994].

For the above reasons (performance, safety, and stability) passive robots have great appeal. A mechanically passive robot operates in continuous time, and naturally provides perceptually smooth performance in response to forces applied to it by a user. Lacking external sources of energy, no hardware or software failure can produce high velocities; kinetic energy is limited to that provided by the user. Lastly, the stability of a mechanically passive device is assured at all frequencies.

\subsection{Background}

While there has been much exploration of active manipulators that can display programmable constraints and that attempt to behave as if passive, we know of only one other physically passive manipulator capable of displaying programmable constraints. In that approach [Troccaz, Lavallee, \& Hellion, 1993; Delnondedieu, Y., \& Troccaz, J. 1995] each joint of a unpowered serial manipulator is free to turn independently, but its angular velocity is limited to a range falling between two limits, enforced by overrunning clutches. A two-dof prototype has been built and a 3-dof prototype is planned.

Also relevant is [Soerdalen, Nakamura, \& Chung, 1994] which concerns nonholonomic planning for active robots. The authors describe an $n$-joint serial robot which can reach any pose in its $n$-dimensional configuration-space, yet possesses only two actuators. Its joints are coupled by $n-1$ nonholonomic devices, and it reaches a desired pose by traversing an intricate path through joint space. The planning of this path is algorithmically equivalent to maneuvering an airport baggage-train of $n$ cars into a desired configuration, solely by controlling the speed of the two drive wheels of the front car.

In our work our intent is not to produce an active manipulator with a minimum number of actuators, but to produce a passive robot which can display programmable constraints. Despite this difference of purpose we use, like Soerdalen et al, $n-1$ nonholonomic devices. These devices differ from those of Soerdalen et al in that they are individually controllable, or "steerable"; section 5 describes their design. Section 4 speculates on why nonholonomic 
devices are essential if one wishes a manipulator to have fewer actuators than the dimensionality of its configuration space, as is the case both in our work and that of Soerdalen et al.

\section{A simple programmable constraint machine (PCM)}

We first describe the concept and construction of a planar 2-dof PCM based on a single rolling wheel, shown in figure 1. This machine, which has been built in prototype, is more fully discussed in [Colgate, Peshkin, Wannasuphoprasit, and Chiou, 1996]. For the purposes of this paper we must introduce it briefly since it is the simplest example of a programmable constraint machine, upon which the other devices described here are based, and which motivates the rotational CVT introduced and analyzed later in this paper.

A PCM is a mechanism which can allow arbitrary full-dof (here, 2-dof) motion of its end-effector as guided by the user in some parts of its workspace. Under computer control, certain parts of the workspace are programmable constraints, of which a simple example is the virtual wall. When the user moves the handle up to a virtual wall, the allowed motion is suddenly reduced in dimensionality.

In figure 1 the device possesses only one degree of freedom when it is in contact with the virtual wall. The shape of the wall may be quite complex and curved, and programmable constraint machines with greater than two degrees of freedom can present programmed constraints of various reduced dimensionalities.

The PCM device travels on $x-y$ rails, where the $x-y$ position of the handle is measured by linear potentiometers on the rails. The device is entirely free to slide on the rails as it is moved by the user. No motors act on it. The rails serve only to restrict its motion to the $x-y$ plane.

An attached wheel rolls on an $x-y$ surface. A motor is attached to the wheel to steer it, i.e. to orient the wheel so that its rolling direction in the $x-y$ plane is under computer control. Note that the motor cannot cause the wheel to roll; only the user can do that, by moving the handle. The steering motor determines the direction in which the wheel can be rolled by the user.

The shaft which steers the wheel is instrumented with strain gauges to measure the lateral force on the wheel, which is the force perpendicular to the direction that the wheel can roll.

The machine operates in two modes. In the "free" mode, away from the virtual wall, the device must appear to the user to provide unconstrained (2-dof) motion. In the "constrained" mode, when the handle is at the virtual wall, the device must appear to the user to have only one degree of freedom: only motion tangentially to the wall is possible.

Note that the motion of the handle is kinematically one degree of freedom at all times, owing to the rolling condition imposed by the wheel. Thus it is the constrained (1-dof) mode which is the more intrinsic mode of this machine, in marked contrast to other schemes for haptic display. To implement the constrained (1-dof) mode, it is only necessary for the $x-y$ position of the handle to be determined by reading the linear potentiometers on the rails, and to use the steering motor to set the wheel at an angle tangential to the virtual wall at the current $x-y$ position. Since the user can only move the handle in such a direction that the wheel rolls, the device appears to contain a physical barrier at the position of the virtual wall.
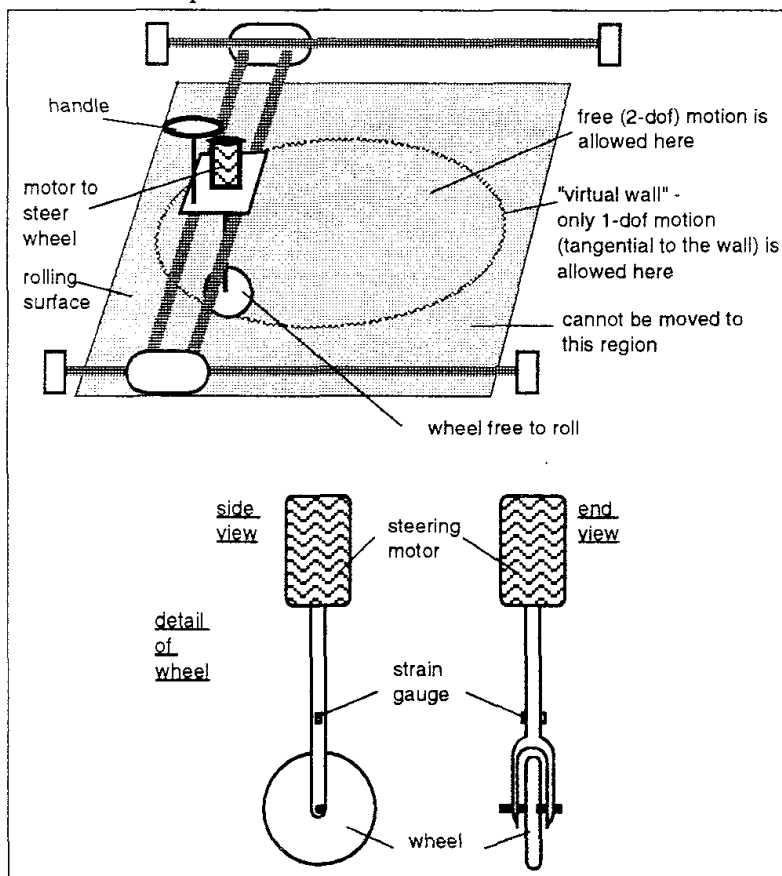

Figure 1. A 2-dof planar PCM. The wheel is free to roll in response to forces applied to the handle by the user. The steering motor sets the available rolling direction. Strain gauges measure lateral forces (perpendicular to the rolling direction.) When in contact with a virtual wall, the wheel is simply steered tangent to the wall. Away from virtual walls, the wheel is steered such as to null lateral forces. It acts as an "electronic caster", and appears to the user to move freely in 2 dimensions.

The free (2-dof) mode must be put into effect whenever the handle is not at a virtual wall, as determined by reading the $x-y$ position. The device remains kinematically 1 -dof, and greater freedom of motion is simulated by steering the wheel so that, to the user, two degrees of freedom appear to be available. To implement the free (2-dof) mode, the strain gauges are read, measuring the lateral force on the wheel (perpendicular to the rolling direction.) The lateral force is nulled by steering the wheel clockwise or counterclockwise. The resulting behavior is that of an "electronic caster", simulating the behavior of the caster wheels typically used on rolling items of furniture to allow free planar motion.

\section{Generalizing to higher degrees of freedom}

The essential distinction between PCMs as introduced here, 
and other routes to haptic display or human-interactive robots, can be expressed compactly. It is that PCMs are kinematically single degree of freedom. This remains true, even when the number of apparent degrees of freedom in the "free" mode of the machine is higher than that of the 2-dof example above. Greater numbers of freedoms are simulated as needed, by steering in response to forces applied by the user to the machine. The 1-dof nature of all PCMs, and the simulation of higher dof, will become clearer in the sequence of examples which follows.

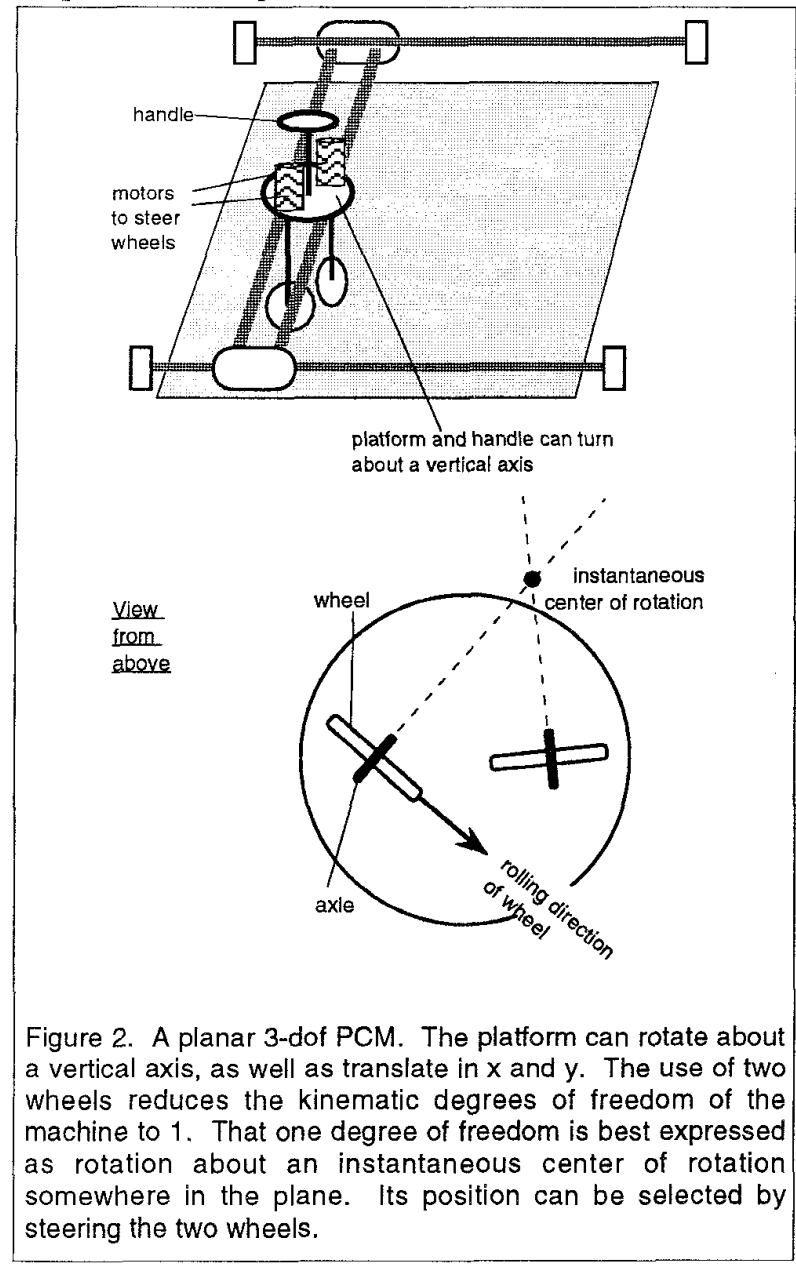

Planar motion can include a rotational axis as well as $\mathrm{x}$ and $y$ translation, totaling 3-dof. A 3-dof PCM thus uses two wheels (as shown in figure 2), reducing the kinematic degrees of freedom to 1. A top view of the platform, to which the two wheels and the handle are attached, is illustrated. Because of the two rolling constraints, the platform can at any moment execute only a single motion, the one which is described by an instantaneous center at the intersection of the axes of the two wheels. By steering the two wheels, the instantaneous center can be positioned anywhere in the plane.

By nulling lateral forces on the two wheels independently, apparent "free" 3-dof motion can be achieved. By positioning the instantaneous center as a function of the 3 -space coordinates of the platform, and ignoring lateral forces, motion is restricted to a programmable 1-dof trajectory through 3-space. The latter behavior corresponds to contact of a planar rigid body with two frictionless walls simultaneously, leaving 1-dof. Between these behaviors lies an intermediate behavior corresponding to contact of a planar rigid body with a 1 -dof wall, with 2-dof motion remaining available to the user.

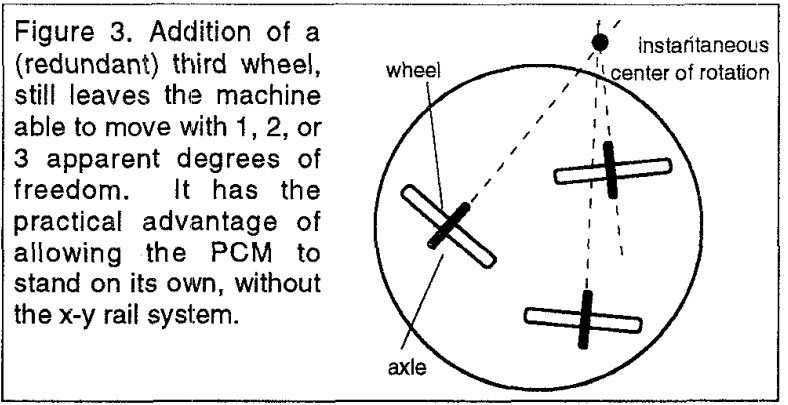

\section{2. A 3-dof spherical PCM}

The PCMs above control planar motion, in 2 or 3 degrees of freedom, by coupling the planar degrees of freedom using rolling wheels. Instead of planar motion, we now consider spherical motion: the three rotations of a sphere about a fixed center.

The PCM shown in figure 4 is a 3-axis joystick or haptic

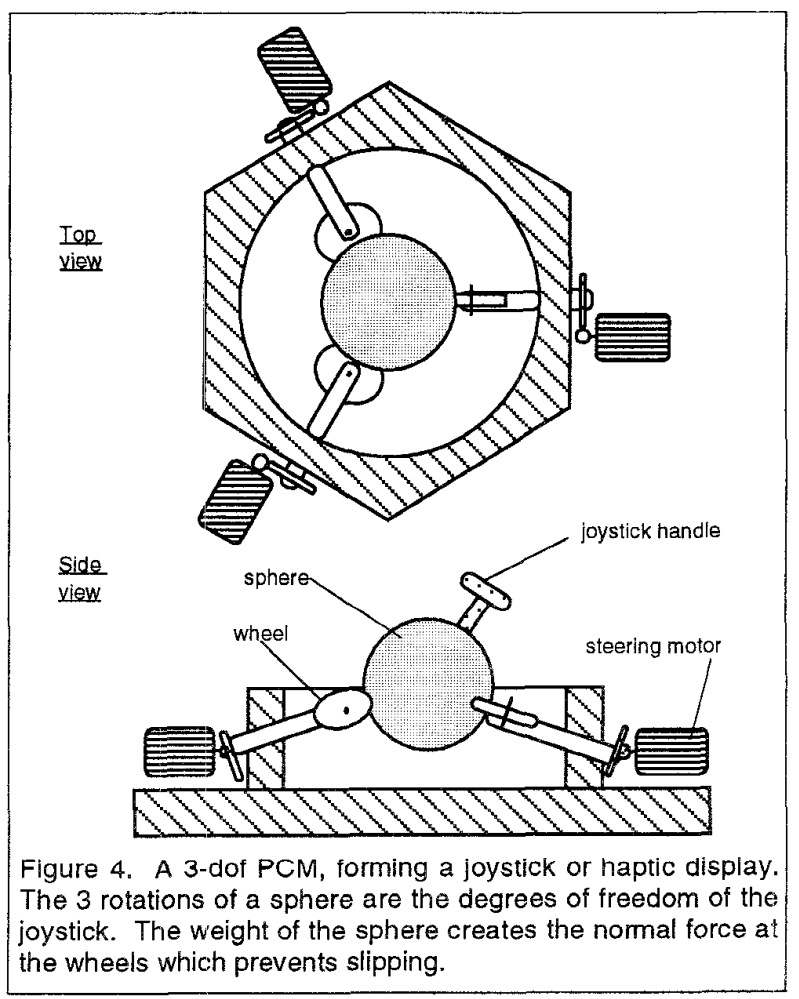


display. The 3 degrees of freedom of the sphere (to which a handle is attached), are reduced to 1 by the use of two wheels. A third wheel is used in order to fully support the sphere without extraneous hardware. The wheels are steered by motors as shown.

\section{The PCM as a nonholonomic power train}

Since a PCM has a single degree of freedom at all times, the whole machine may be thought of as a complex transmission, or "power train". The elementary CVTs (e.g. wheels) which couple the degrees of freedom of the machine serve to transmit power between those degrees of freedom. It is this feature - that all the degrees of freedom can communicate power to one another with little loss - that makes the user's manually supplied power sufficient to drive the robot, and makes possible its passive design.

A CVT couples two velocities by a ratio which is adjustable. In the case of the rolling wheel, which is the only CVT we have mentioned so far, we consider it to be a device which couples the two translational velocities of its center, $v_{x}$ and $v y .$. In figure 1 , The two degrees of freedom of the device are $v^{\prime} x$ and $v y$, and the rolling constraint imposed by the wheel is $v_{y} / \gamma_{x}=\tan (\alpha)$. By controlling the steering angle $\alpha$, we can vary the transmission ratio relating $v x$ and $y y$ continuously through the entire range $-\infty$ through $+\infty$.

There are many existing CVT designs which permit a continuously variable transmission ratio over a limited range. The rolling wheel, and its rotational analog which we will introduce in the next section, are special in that they allow a full range of transmission ratios of both signs.

As we have mentioned. PCMs are kinematically 1-dof. At every moment the velocities of all of the moving components of a PCM are held in proportion by CVTs. While the velocities are held in proportion, the displacements of the components are not likewise coupled, because the velocity ratio is variable over time.

Coupled velocities in the absence of coupled displacements is the defining characteristic of nonholonomic constraints. of which the rolling wheel is the canonical example. All CVTs are nonholonomic. CVT nonholonomy is responsible for allowing a PCM to reach all parts of its high-dimensional configuration space, while having at any instant just one degree of freedom. because configuration space relates to displacements, while degrees of freedom relates to velocities. Power transmission relates to velocities, which is why a PCM can transmit power efficiently amongst its parts.

\section{The rotational analog of the wheel (thought of as a CVT)}

Recall that the rolling wheel is a translational CVT: it eouples the ranslational velocities $x$ and $y$ of its center. with $r / x$ equal to a adjustable transmission ratio. The whel in its capacity as a CVT may be expected to have a rotational analog.
The elementary rotational CVT would couple two shafts, with angular velocity $\omega_{1}$ or $\omega_{2}$, such that $\omega_{2} / \omega_{1}=c$, with $c$ being an adjustable transmission ratio. A rotational CVT with this behavior is developed below. To the best of our knowledge it is a novel kinematic mechanism. We develop it first in an easily explained but mechanically sub-optimal form, and then in the mechanically preferable form which is currently under construction.

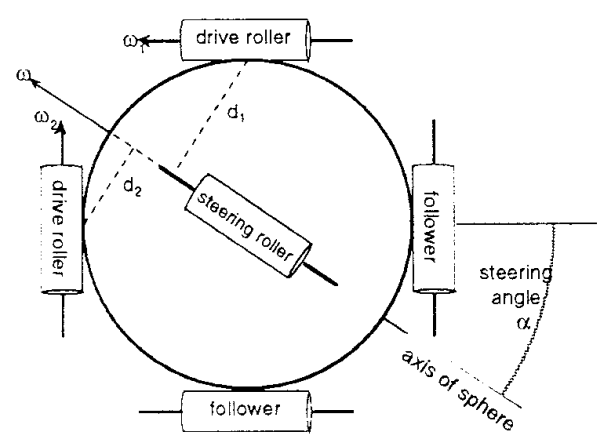

Figure 5. The rotational CVT, analogous to the rolling wheel which is a translational CVT. Six rollers confine a sphere. The angular velocities of the two drive rollers are held in the proportion $\omega_{2} / \omega_{1}=\tan \alpha$. by the steering rollers.

The simpler form is shown in figure 5 . It consists of a sphere caged by six rollers, with the rollers arranged as if on the faces of a cube surrounding the sphere. Each of the six rollers is pressed in toward the center of the sphere by an externally applied force $F_{\text {preload. The force }} F_{\text {preload }}$ serves to keep each of the rollers in rolling contact with the sphere. We do not show the frame which holds the rollers, or the bearings which allow the rollers to turn, or the springs which supply the force $F_{\text {preloat }}$.

Two of the rollers are considered drive rollers. These are the ones that interface to other parts of a machine that incorporates the CVT. These drive rollers have angular velocities $\omega_{1}$ and $\omega_{2}$. Two other rollers, diametrically opposite the drive rollers, are followers. They serve only to confine the sphere and to apply the force Freica. They rotate with angular velocity $\omega_{1}$ and $\omega_{2}$ also, but this rotation is not used. These four rollers (two drive rollers and two followers) have axes of rotation that all lie in a single plane, and this plane passes through the center of the sphere.

The remaining two rollers are steering rollers, at the top and bottom of the sphere. The steering rollers can turn freely on their axes. Conly the top steering roller can be seen in the figure, as the bottom steering roller is hidden beneath the sphere. The bottom steering roller is oriented identically.) Unlike the drive rollers and followers, the axis of the steering rollers is adjustable. The angle that the axis of the steering roller forms with the horizontal is the steering angle o. The mechanism which allows us to vary the steering angle. and which keeps the steering angles of the top and bottom steering rollers in agreement, is not shown. 
Before attempting to visualize the motion of the sphere, it is worthwhile to review the condition under which rolling (as opposed to sliding) occurs between two rotating rigid bodies in contact. The condition is that the axes of the two bodies are coplanar. Their axes do not need to be parallel: the axes of two bevel gears (which are in rolling contact) are coplanar but not parallel. If, on the other hand, the axes of the two bodies are skew, then sliding occurs, or, if the coefficient of friction is adequate, motion is prevented. The rotational CVT requires a sufficient force $F_{\text {preload }}$ and coefficient of friction $\mu$ to prevent sliding.

The kinematics of the rotational CVT may now be understood as follows. Consider all possible axes of rotation of the sphere. The sphere must be in rolling contact with all six rollers if it is to move at all. Since the center of the sphere is stationary, the sphere's axis of rotation must pass through its center. Rolling contact with a given roller requires that the axis of the sphere lies in the plane containing the axis of the roller, and also passing through the center of the sphere. Each roller forms such a plane. (The planes for the followers and the bottom steering roller can be ignored, by symmetry.)

The axis of rotation of the sphere must be the intersection of the three planes demanded by the two drive rollers and one steering roller. Such an axis exists: it is in the plane of the paper, passing through the center of the sphere, and parallel to the axis of the steering roller. It is labeled "axis of sphere" in Figure 5.

Now consider the linear velocities of the points of contact between the drive rollers and the sphere. If the radius of the rollers is $R_{\text {roller }}$, the velocities of these points of contact are $\omega_{1} R_{\text {roller }}$ and $-\omega_{2} R_{\text {roller, }}$, perpendicular to the paper. If the angular velocity of the sphere (about its axis identified above) is $\omega$, and the distances from that axis to the points of contact are $d_{1}$ and $d_{2}$, then the velocity of the points of contact can also be computed as $d_{1} \omega$ and $-d_{2} \omega$. Equating $\omega_{1} R_{\text {roller }}=d_{1} \omega$ and $-\omega_{2} R_{\text {roller }}=-d_{2} \omega$ we find $\omega_{2} / \omega_{1}=d_{2} / d_{1}$. Note from the geometry that $d_{2} / d_{1}=\tan (\alpha)$. Thus we have established an adjustable transmission ratio between the angular velocities of the two drive rollers

$$
\frac{\omega_{2}}{\omega_{1}}=\tan \alpha
$$

where $\alpha$ can be interpreted as the steering angle, just as for a rolling wheel.

\section{I A tetrahedral rotational CVT}

Figure 6 shows a modified rotational CVT, which has many practical advantages. Its principle of operation is the same as in figure 5 , but it requires only four rollers instead of six. (Four is the minimum number of point contacts needed to confine a sphere.) The two follower rollers are thus eliminated. The rollers contact the sphere at four points describing the corners of a tetrahedron. It is not a regular tetrahedron, but rather a stretched one, such that the angle subtended by the points of contact of either pair of rollers with the center of the sphere is 90 degrees. This facilitates machining. (For a regular tetrahedron this angle would be 108 degrees).

The rollers no longer need to be independently preloaded. Instead, a rigid frame holds the two drive rollers, and another rigid frame holds the two steering rollers. These two frames can be simply drawn together by a spring, which will apply the same force $F_{\text {preload }}$ to all four contacts.

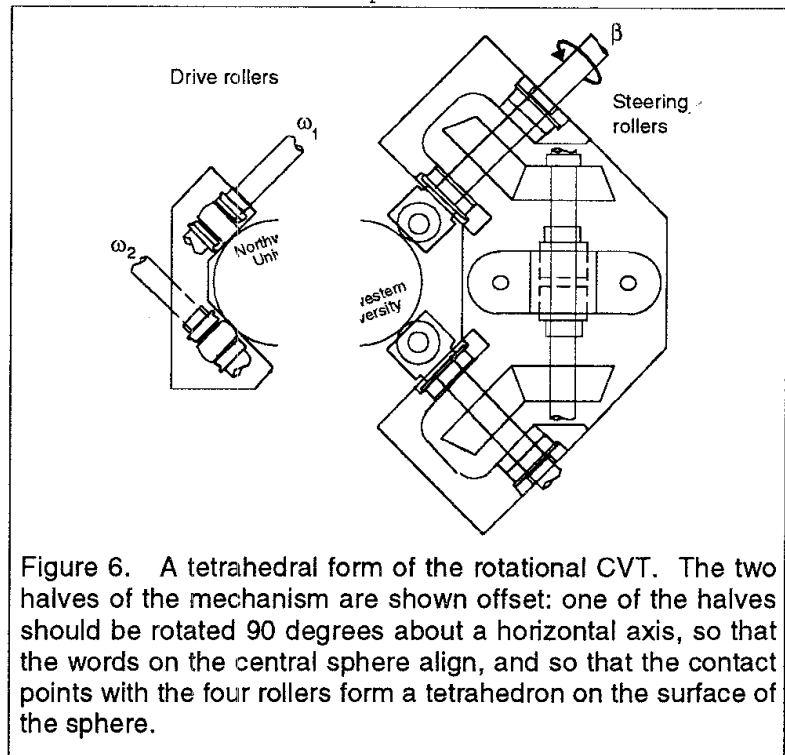

Figure 6 also shows the gear mechanism which causes the two steering rollers to stay aligned. The axes of the two steering rollers are rotated in opposite directions by this mechanism: one turns clockwise while the other turns counterclockwise.

Visualizing the axis of rotation of the sphere is more difficult for the tetrahedral arrangement than for the cubic above. Use of the coplanarity condition for rolling is essential. The axes of the drive rollers are perpendicular and coplanar, just as they were in the cubic arrangement. The axes of the two steering rollers are not parallel, as they were in the cubic arrangement. They are in fact coplanar, but the plane that they share does not in general contain the center of the sphere. Rather, the two distinct planes formed by the axis of each steering roller with the center of the sphere intersect one another, and that line of intersection is the axis of rotation of the sphere. It lies in the plane of the drive rollers.

Careful geometry yields the transmission ratio

$$
\frac{\omega_{2}}{\omega_{1}}=\frac{-\cos 45 \cot \beta-1}{-\cos 45 \cot \beta+1}
$$

where $\beta$ is the angle to which the axes of the steering rollers have been turned relative to the configuration shown in the figure. This transmission ratio assumes a full range of values $(-\infty$ through $+\infty)$ as the steering angle $\beta$ is changed. 


\subsection{Serial robot architectures using the rotational CVT}

While a translational CVT (the rolling wheel) was useful in PCMs with parallel architectures as described in section 4, a rotational CVT is useful in serial architectures, such as conventional serial robots. One may imagine coupling the revolute joints of any serial robot architecture with rotational CVTs, and removing the motors, yielding a PCM. An $n$-joint serial revolute robot requires $n$ - 1 CVTs, each one coupling two consecutive joints. Figure 7 shows a two-joint manipulator which uses a single rotational CVT. The extension to greater numbers of joints is straightforward.

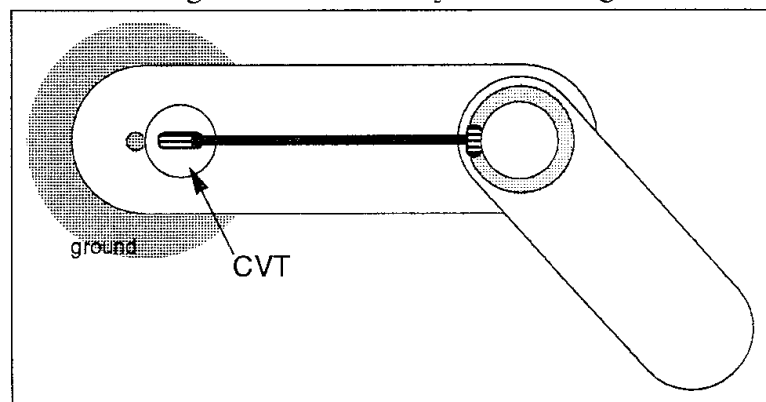

Figure 7. A serial PCM using a rotational CVT. The rotation of joint 1 is coupled to one of the drive rollers of the CVT, and the rotation of joint 2 is coupled via an extension rod and bevel gears, to the other drive roller of the CVT. The steering rollers of the CVT are not shown. A similar arrangement would couple the rotation of joint 2 to a third joint, etc.

\section{Discussion}

This paper and its companion paper [Colgate, Peshkin, Wannasuphoprasit, and Chiou, 1996] introduce a new approach to haptic or robotic devices for interaction with people. This paper has addressed primarily the kinematic aspects, while its companion focuses on the control aspects.

The crucial distinction between the "programmable constraint machine" approach described here and prior approaches to haptic devices should now be very clear. In most prior approaches, the mechanism has as many kinematic degrees of freedom as the resulting device is expected to exhibit, and its joints are driven by motors. If the motors are inactive the user can move the device freely. When it is desired to reduce the number of degrees of freedom (in order to exhibit a virtual constraint surface), the motors work to couple the motion of the joints, so that the user's ability to move the device is constrained.

In contrast, in the PCM concept, the mechanism has at most one kinematic degree of freedom, because its joint velocities are mechanically held in proportion by CVTs. When the motors that adjust these CVTs are inactive, the user can only move the manipulator along a one-dimensional curve through space. When it is desired to increase the number of degrees of freedom (i.e. when positioned away from software constraint surfaces), the motors work to adjust the CVTs' transmission ratios so that the device complies with the user's desired motions.

The intrinsic mode of a PCM is the constraint mode, and the free mode is achieved through servo control. Haptic devices in which the joints are driven by motors have an intrinsic mode which is free motion, and achieve their constraint modes through servo control.

Among the consequences of unpowered joints is passivity: all the power that moves the device originates in the user's muscles. Safety issues associated with the use of motors strong enough to constrain human motion are avoided. Stability issues are reduced.

We have identified the CVT (continuously variable transmission) as the fundamental kinematic element of PCMs, and nonholonomic constraint as the CVT's essential contribution. The fundamental control element is the electronic-caster behavior, which makes the CVT responsive to forces perpendicular to its constraint surface. That behavior is addressed in our companion paper, and has been demonstrated on a prototype device.

Most of the kinematic architectures described in this paper have used as a CVT the rolling wheel, which is the canonical example of a nonholonomic constraint. Because CVTs appear to be so important to PCMs, we have proposed an analog to the rolling wheel, the rotational CVT, which couples angular velocities in the same way that the rolling wheel couples translational velocities. This device is currently under construction. To our knowledge it is a novel device.

\section{Acknowledgments}

This work was supported by grants from General Motors and the Whitaker Foundation, and by fellowship support of the Illinois Minority Graduate Incentive Program.

\section{References}

Colgate, J. E., \& Brown, J. M. (1994). Factors Affecting the Z-width of a Haptic Display. IEEE International Conference on Robotics and Automation, (pp. 3205-10). San Diego, CA

Colgate, J. E., Peshkin, M. A., Wannasuphoprasit, W., and Chiou, Y C. (1996). Nonholonomic Haptic Devices. IEEE International Conference on Robotics and Automation, Minneapolis, MN

Soerdalen, O. J., Nakamura, Y., \& Chung, W. J. (1994). Design of a nonholonomic Manipulator. IEEE International Conference on Robotics and Automation, San Diego, CA

Troccaz, J., Lavallee, S., \& Hellion, E.. (1993). Padyc: A passive arm with dynamic constraints. Int. Conf. on Advanced Robotics, pp. 361-366, Tokyo, November 1993, JIRA

Delnondedieu, Y., \& Troccaz, J. (1995). PADyC: a Passive Arm with Dynamic Constraints. A prototype with two degrees of freedom. Medical Robotics and Computer Assisted Surgery pp. 173-180, Baltimore, MD, November 1995, Johns Hopkins \& Carnegie Mellon University 